I do not wish to be undersiood as recommending the bran biscuits which have been advertised as mine (although with all their defects they have assisted in saving many lives), but a cake or loaf made of bran-thoroughly washed, ground, and sifted, according to my directions.

My formula has been altered by the makers and vendors into a biscuit adapted for long keeping; but biscuits are necessarily hard and dry, and not very manageable (especially if over-baked); whilst the cake is soft and so palatable that I am sometimes obliged to check patients as to the quantity.

What I recommend is that, in hospitals or in private cases where the necessity for the diet is likely to continue, a proper mill should be purchased, or the powder ready prepared be obtained instead of biscuits; the manufacture of the cake or loaf from the powder is so simple that it requires no skill, and involves very little time or trouble. It should be remarked, however, that it will be useless to procure a mill unless the bran-washing can be carefully superintended, and the labour of grinding and sifting accomplished. With management, however, I am of opinion that in hospitals the manual labour could, in many instances, be performed by the patients themselves, and they might be supplied with the bran powder after their discharge-a thing of no small consequence where the necessity for a restricted diet is so often permanent. It may be observed, moreover, that bran in its natural state is so cheap that the expense of a mill is very soon saved.

Before closing these remarks I may add, that I have lately tried with success a modification of the formula already alluded to; it has the advantage, where economy is an object, or the stomach does not well bear butter; and, in many instances, it is useful as a change.*

Did your space permit, these remarks might be extended by observations on the other substitutes for bread which have been proposed or attempted; but this would lead me too far: suffice it to say, that all other plans with which I am acquainted have failed. The gluten bread, if so prepared as to be at all palatable, still retains a considerable quantity of starch; and ordinary bread, in whatever form, unless so carbonized as to be unfit for food, is liable to the same objection.

I shall not, on the present occasion, go into the question of the best beverages for the diabetic (a subject which now occupies much of my attention), or the best medical treatment; these matters must be reserved for some future opportunity.

I am, Sir, your obedient servant,

Compton-terrace, Islington, March, 1861. JoHN M. CAMPLIN, M.D.

\section{COLD WATER AND CONSERVATIVE SURGERY.}

\section{To the Editor of THE LaNCET}

SrR,-Perceiving in Trre LANCET of the 16th instant a paragraph with the above heading, in reference to a statement in the Medical Gazette of Oporto, as to wounded limbs having been saved by immersion in cold water, $\mathrm{I}$ can add my testimony to the fact from having had my right thigh fractured by a musket shot at the battle of Toulouse; and having been recommended by the first surgeon who saw it to put the $\operatorname{limb}$ into a running stream close by, where it remained for several hours, until the battle was over, and the inflammation had subsided in a great degree. Mr. Guthrie, who afterwards at tended me, told me that he considered the femoral artery had been cut, and that if he had seen it in time he would most probably have amputated the limb.

I have known several instances during the late Peninsular War of limbs having been thus saved, which so far corroborates the statement of M. Pereira da Fonseca, to which you allude; so that the cold-water cure is not of so recent a date as the disciples of Preissnitz seem to imagine.

March, $1 \varepsilon 61$. I am, Sir, your obedient servant JoHN ScotT LIILIE.

\section{DR. PROSSER JAMES ON "SORE-THROAT."} To the Editor of THE LANCET.

Srr, - In your last number the reviewer of my book on "Sore-throat" intimates that he would "be glad to learn the names of the teachers" who tell "students to open the jugular veins of infants" sufiering from croup.

That I have not exaggerated the activity of the practice long advised in this disease could be shawn by quotations from

* Prepared bran, four ounces, three eggs, about twelve ounces of milk, a little spice and salt, to be mixed and put into a basin (previously well buttered) bake for about an hour. The loaf may be cut into slices, and toasted when wanted, or, after slicing, be re-baked and kept in the form of rusks.

the majority of writers upon it; but as I can scarcely expect you to devote your pages to the proof of what is well known to every tyro, I will only ask you to refer your critic to a pas. sage from one of the most moderate of living teachers. Dr. West says:-

"The abstraction of blood and the administration of tartar emetic are the two measures on which your main reliance must be placed, and you must bleed largely and give tartar emetic freely. ..... I have never met with an exception to the rule which prescribes the free abstraction of blood in every case of severe idiopathic croup when seen at an early period....... Even in young children local depletion forms in these cases but a poor substitute. ...... Bleeding from the jugular vein is preferable under these circumstances to venesection in the arm, since the latter often fails in children under three years old; and the blood never flows so freely as when taken from the jugular vein."-Lectures on Diseases of Infancy and Childhood, third edition, pp. 297-S.

In the same work will be found (p. 594) a reference to Dr. Kennedy's cases of sudden death from sloughing of the neck, involving the large vessels. If it be so "very questionable" whether this process in the throat may "implicate the carotid," what does the reviewer think of the following statement to be found in "Druitt's Surgeons' Vade-Mecum"?

" Ulceration is liable to affect the pharynx at the level of the cricoid cartilage, or the cesophagus lower down. ..... The consequences are always the same: exhaustion from the irritation of the disease and from the inability to swallow, or possibly a more speedy death from perforation of the aorta (!) or of the pleura.

In summing up all that my book tells about the laryngoscope, your reviewer omits-Ulcerated Sore-throat; Ulceration and Polypus of the Pharynx; Aphonia, Hoarseness, Acute Laryngitis, and Hysterical Simulations; Chronic Laryngitis, Laryngeal Phthisis, and Tumours pressing on Larynx or Trachea, Follicular Ulceration of Epiglottis; and Nervous Sore-throat: under each of which I allude to the instrument. Further, the laryngoscopic appearances are stated in several cases in the Appendix, to which a reference is given at p. 30 , from which your reviewer quotes.

In saying that I give " aconite in very small doses," your reviewer should have added that I repeat them until they produce a sensible effect-deeming this safer than prescribing a full dose at once.

I should be very sorry to endeavour to controvert anyone's opinion of my writing, but facts differ widely from opinions; and it is only to correct errors in matters of fact that $I$ have written this letter.

Finsbury-square, March 19th, 1861. I am, Sir, yours, \&c.

M. Prosser James, MrD., Physician to the City and to the Metropolitan

\section{THE ROMAN BATH OR THE LAMP BATH?}

\section{To the Editor of The LANCET.}

SIR, - In a letter published in your journal of the 9 th instant, I observe Dr. Lockhart Robertson to remark that he has never heard that the human skin possesses any respiratory function, except on the authority of pamphleteers on the koman and Turkish bath. I will leave it to those gentlemen whom Dr. Robertson has invited to solve the point-viz., "our teachers of physiology"- to supply him with a full reply; but in the meantime I would call his attention to the remarks of one physiologist on the subject-viz., Dr. Carpenter, who, in his "Principles of Physiology," says, -

"It has been ascertained, not unfrequently, that the livid tint of skin which supervenes in asphyxia, owing to the nonarterialization of blood in the lungs, has given place, after death, to the fresh hue of health, owing to the reddening of the blood in the cutaneous capillaries by the action of the atmosphere upon them; and it does not seem improbable that, in cases of obstruction to the due action of the lungs, the exhalation of carbonic acid through the skin may undergo considerable increase. Moreover, there is evidence that the interchange of gases between the air and the blood, through the skin, has an important share in keeping up the temperature of the body."

For my own part, I do not think there is room to doubt that the human skin possesses certain respiratory functions, though of course, in a much more limited degree than in the batrachia. It is true, however, that in far the greater proportion of cases where a medical man is consulted, the skin is not in a condition to perform its respiratory function: and how can it possibly be 